\title{
Hydroxychloroquine treatment for primary Sjögren's syndrome: its effect on salivary and serum inflammatory markers
}

\author{
Moshe Tishler, Ilana Yaron, Idrit Shirazi, Michael Yaron
}

\begin{abstract}
Objective-To evaluate the effect of hydroxychloroquine treatment on interleukin 6 (IL6), hyaluronic acid (HA), and soluble interleukin 2 receptor (sIL2R) concentrations in the saliva and serum of patients with primary Sjögren's syndrome (SS).

Methods-Fourteen SS patients treated with hydroxychloroquine $200 \mathrm{mg} / \mathrm{day}$ for 12 months were investigated in an open prospective study. Clinical parameters of efficacy and routine biochemical and haematological data to assess drug safety and tolerability were determined every three months. Salivary and serum IL6, sIL2R, and $H A$ values were determined at study entry, 6 and 12 months, using ELISA and radiometric assays.
\end{abstract}

Results-After hydroxychloroquine treatment, salivary IL6 concentrations decreased from $13.2(1.2)$ to $7.3(1.1) \mathrm{pg} / \mathrm{ml}$ $($ mean $($ SEM) $)(p<0.0001)$. Similarly, salivary HA concentrations were also reduced from $577.8(120)$ to $200(34) \mathrm{ng} / \mathrm{ml}$ (mean (SEM) $(p<0.003)$. Serum IL6 concentrations decreased from $5.4(0.6)$ to 2.9 (0.2) $\mathrm{pg} / \mathrm{ml}$ (mean (SEM) $(\mathrm{p}<0.001)$, while serum HA concentrations remained unchanged. No change has been detected in salivary or serum sIL2R concentrations after 12 months of treatment with hydroxychloroquine. Treatment also resulted in significant reduction in erythrocyte sedimentation rate, serum $\gamma$ globulin, and $\mathrm{C}$ reactive protein values while only partial clinical improvement was noted in some patients. A more pronounced decrease of salivary IL6 and HA levels was found in the two patients in whom a reduction in the swelling of the parotid gland was noted.

Conclusion-In this open label study of hydroxychloroquine treatment for SS a significant reduction of some salivary inflammatory markers was seen at the end of 12 months. Although during the treatment period only a partial clinical effect could be noted, the findings suggest that a double blind controlled study of hydroxychloroquine in SS is indicated.

(Ann Rheum Dis 1999;58:253-256)

Primary Sjögren's syndrome (SS) is a systemic autoimmune disease characterised by dryness of eyes and mouth. The histopathological picture is associated with lymphocytic infiltra- tion of salivary and lacrimal glands, mostly $\mathrm{CD}_{4}{ }^{+} \mathrm{T}$ cells. ${ }^{1}$ Because of the generally benign character of the disease it is mainly managed by symptomatic measures only. Hydroxychloroquine has been found to have a beneficial effect in rheumatoid arthritis and systemic lupus erythematosus. Although its exact mechanism of action is not fully understood, it has been suggested that it is caused by interference with macrophage processing of antigens, resulting in interaction with $\mathrm{T}$ cell activation. As $\mathrm{T}$ cell derived cytokines are required for $\mathrm{B}$ cells to switch from IgM to IgG synthesis, the assumption is that hydroxychloroquine would decrease IgG hyperglobulinaemia in patients. This led investigators to use this drug in SS with the belief that reduction of the inflammatory process taking place in the exocrine glands can result in symptomatic improvement of dryness. Indeed, the effect of hydroxychloroquine on patients with SS has been investigated in several studies. ${ }^{2-4}$ The outcome of all reports was that although a significant decrease in IgG and erythrocyte sedimentation rate (ESR) was achieved as expected, no beneficial clinical effect with regard to mucosal symptoms and signs of SS could be demonstrated.

Assessing the inflammatory component of the salivary glands in SS patients is complicated and has been a matter of debate. Recently, we have proposed that salivary analysis could be an easy and applicable tool for assessing salivary involvement in SS. ${ }^{5}$ Furthermore, interleukin 6 (IL6) and hyaluronic acid (HA) have been shown to be specifically increased in the saliva of SS patients. ${ }^{67}$ As the anti-inflammatory effect of hydroxychloroquine has been shown in serum samples of SS patients, we speculated that a similar effect might be expected on salivary inflammatory markers (IL6, sIL2R, and HA).

\section{Methods}

Fourteen patients diagnosed as having primary SS according to the EC proposed criteria ${ }^{8}$ were included in the study. These patients are part of a larger cohort of SS patients followed up routinely in the Sjögren's clinic at the Department of Rheumatology, Tel Aviv Medical Center. Exclusion criteria for this study included treatment with hydroxychloroquine, corticosteroids, non-steroidal anti-inflammatory drugs (NSAIDs) or immunosuppressive drugs in the three months before the study. No patient had any ophthalmological contraindications for the use of hydroxychloroquine nor any apparent oral infection. All patients were women, their 
Table 1 Effect of hydroxychloroquine treatment on various laboratory parameters

\begin{tabular}{llll}
\hline & Time (0) & Time (6 months) & Time (12 months) \\
\hline ESR (mm 1st h) & $43.1(18.6)$ & $37.2(15.1)$ & $27.2(12.4)^{\star}$ \\
CRP (mg/dl) & $3.4(1.1)$ & $3.1(1.2)$ & $2.1(0.7)^{\star}$ \\
IgG (mg/dl) & $2350(460)$ & $2190(450)$ & $1560(310)^{\star}$ \\
IgM (mg/dl) & $320(45)$ & $305(41)$ & $295(50)$ \\
IgA (mg/dl) & $300(25)$ & $295(30)$ & $280(35)$ \\
Salivary flow rate ml/min & $0.07(0.01)$ & $\mathrm{ND}$ & $0.08(0.02)$ \\
Serum IL6 (pg/ml) & $5.4(0.6)$ & $4.5(0.8)$ & $2.9(0.2) \dagger$ \\
Salivary IL6 & $13.2(1.2)$ & $8.7(1.6)^{\star}$ & $7.3(1.1)^{\star}$ \\
Serum HA $(\mu \mathrm{g} / \mathrm{l})$ & $142.4(90.7)$ & $130.8(97)$ & $106(69)$ \\
Salivary HA & $577.8(120)$ & $381.6(115.2)$ & $200.2(34) \dagger$ \\
Serum sIL-2R (pg/ml) & $1481.4(163.5)$ & $1471(146)$ & $1463.6(125.9)$ \\
Salivary sIL-2R & $74.3(6.8)$ & $68.1(5.8)$ & $65.2(4.7)$ \\
\hline
\end{tabular}

$\mathrm{ND}=$ Not done. ${ }^{\star} \mathrm{p}<0.05 . \mathrm{tp}<0.001$. Data are presented as mean $(\mathrm{SEM})$.

mean (SD) age was $58(10.3)$ and their mean (SD) disease duration 4.2 (2.3) years. Patients were treated with Plaquenil (hydroxychloroquine) $200 \mathrm{mg}$ daily for 12 months. Clinical parameters of efficacy were determined every three months and included: (a) a special questionnaire using a visual analogue scale for the presence and severity of the following symptoms: dryness of mouth, swelling of the salivary glands, dryness of eyes, fatigue, arthralgia, and sleeping problems, and (b) physical examination for the signs of parotid swelling, lymphadenopathy, and synovitis.

Biochemical parameters, determined at study entry and after 6 and 12 months, included ESR, serum $C$ reactive protein (CRP) concentrations, serum protein electrophoresis as well as routine haematological and biochemical testing to assess drug safety and tolerability.

IL6, SIL2R, AND HA ASSAYS

IL6, sIL2R, and HA concentrations in serum and saliva were determined at study entry, and at 6 and 12 months, as previously described. ${ }^{7}$ Briefly, saliva and blood samples were collected from patients at the same visit. Whole saliva was collected by spitting for 10 minutes into a test tube and its volume was recorded. Samples of serum and saliva were immediately frozen after centrifugation and stored at $-20^{\circ} \mathrm{C}$ until processed. The assays were carried out blindly

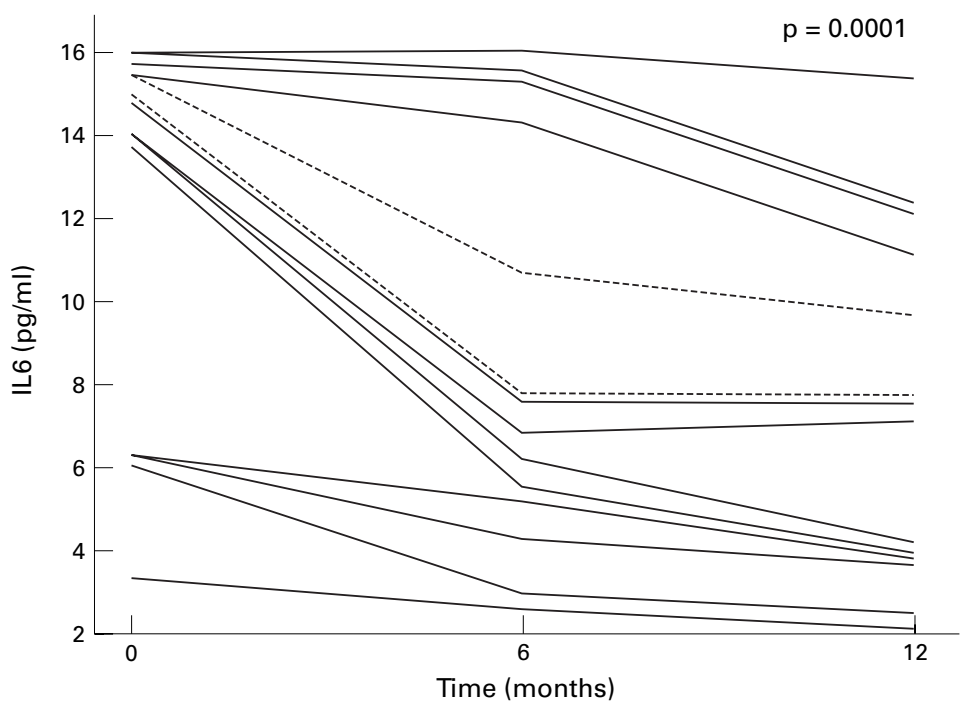

Figure 1 Salivary IL6 concentrations after hydroxychloroquine treatment. Dashed lines = patients with decrease in parotid swelling. with respect to the timing of collection. HA concentrations of blood and saliva were determined using a radiometric assay (Kabi Pharmacia, Uppsala, Sweden), IL6 concentrations of serum and saliva were evaluated using a quantitative ELISA kit (Quantikine High Sensitivity $\mathrm{R}$ and D Systems, Inc, Minneapolis, $\mathrm{MN}$ ) while concentrations of sIL2R in saliva and serum were determined by a quantitative ELISA kit (Quantikine High Sensitivity R and D Systems, Inc, Minneapolis, MN).

\section{STATISTICAL ANALYSIS}

The effects of treatment with hydroxychloroquine on the various parameters tested were analysed by paired $t$ test comparing differences between baseline and 12 month values.

Correlations between the various inflammatory markers and between their concentrations and disease variables were determined using the Pearson product moment correlations.

\section{Results}

All 14 patients completed the study and no patient required addition of corticosteroids or immunosuppressive drugs during the follow up period. No side effects were recorded during treatment.

\section{CLINICAL SYMPTOMS}

Two patients reported improvement in the feeling of xerostomia after 12 months of treatment. Swelling of the parotid gland was significantly reduced in two patients and arthralgia was improved in four patients. No clear improvement was noted with respect to symptoms of dryness or sandy feeling of the eyes, recurrent inflammation of the eyes or fatigue; nevertheless, sleep disorders were improved in two patients.

LABORATORY VALUES

After hydroxychloroquine treatment a statistically significant improvement in ESR, serum CRP, and serum $\gamma$ globulin values $(\mathrm{p}<0.05)$ was seen (table 1). No change was detected in other haematological, biochemical or serological tests. There were no changes in salivary flow rates during the follow up period.

\section{IL6, HA, AND SIL2R CONCENTRATIONS}

Significant reduction was detected in salivary HA and IL6 concentrations at 12 months when compared with baseline values (figs 1,2). Salivary IL6 concentrations decreased from 13.2 (1.2) $\mathrm{pg} / \mathrm{ml}$ to 7.3 (1.1) $\mathrm{pg} / \mathrm{ml}$ (mean (SEM); $\mathrm{p}<0.0001)$. Similarly, salivary HA concentrations were also reduced from 577.8 (120) $\mathrm{ng} / \mathrm{ml}$ to 200.2 (34) $\mathrm{ng} / \mathrm{ml}$ (mean $(\mathrm{SEM}))(\mathrm{p}<0.003)$. Serum IL6 concentrations were significantly reduced, decreasing from $5.4(0.6)$ at baseline to $2.9(0.2) \mathrm{pg} / \mathrm{ml}$ at 12 months (mean $(\mathrm{SEM}))(\mathrm{p}<0.001)$, while serum HA concentrations remained unchanged during the follow up period. No change was detected in salivary or serum sIL2R concentrations after 12 months of treatment with hydroxychloroquine (table 1). Values of the various markers remained 


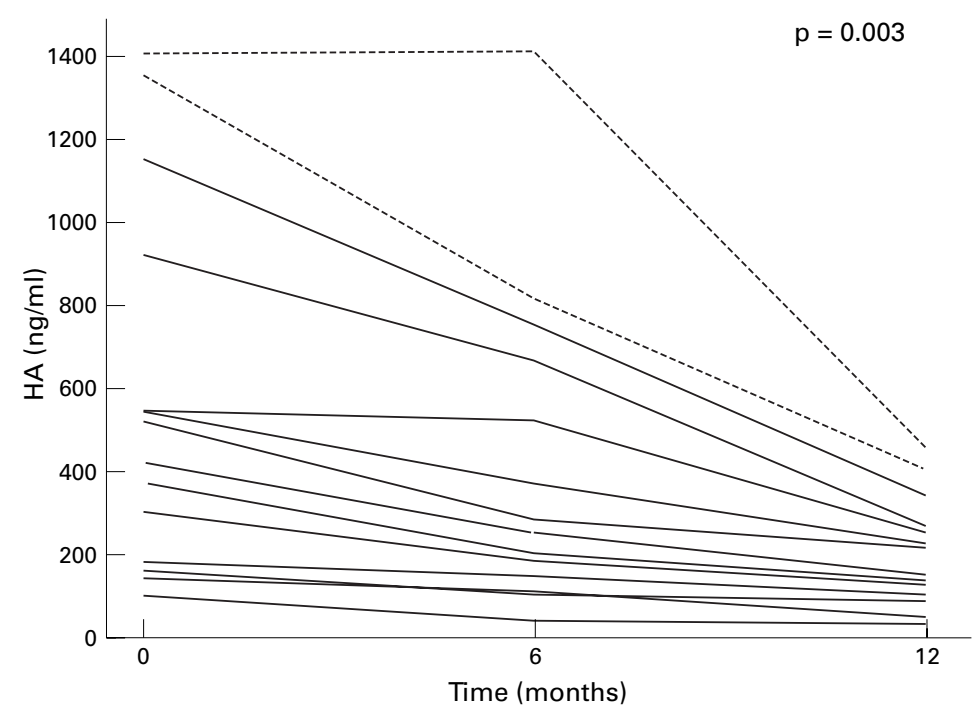

Figure 2 Salivary HA concentrations after hydroxychloroquine treatment. Dashed lines = patients with decrease in parotid swelling.

unchanged when correlated with the amount of saliva obtained for testing (by determining the output of IL6, HA, and sIL2R as concentration times flow rate).

CORRELATIONS

The decrease in salivary IL6 concentrations highly correlated with that of salivary HA concentrations for each patient tested $(r=0.824$, p $<0.001)$ and that of ESR $(r=0.628, \mathrm{p}<0.05)$. The two patients in whom a decrease in the swelling of the parotid gland was noted were found to have a statistically significant decrease in salivary IL6 and HA concentrations as compared with the other patients in the study group $(\mathrm{p}<0.01)$. No other correlations concerning clinical improvement of arthralgia, sleep disorders or xerostomia could be detected.

\section{Discussion}

The use of hydroxychloroquine in SS has been studied for the past decade by several investigators..$^{2-4}$ The conclusions of all studies, whether retrospective, prospective or double blind, were quite similar and showed no significant clinical beneficial effect. Nevertheless, in most of them a decrease in some inflammatory parameters, such as ESR and IgG was noted. ${ }^{2}$ Our study, which is a prospective open trial, supports the results of earlier studies, but offers us the possibility of opening a window to a unique mechanism of action of hydroxychloroquine in SS. Although after 12 months of treatment only mild beneficial clinical effects were noted, such as improvement of arthralgias and parotid swelling, its anti-inflammatory activity is promising. While almost all other studies looked for the effect of the drug on serum inflammatory markers, we preferred to focus on the specific action on the salivary glands by evaluating salivary markers such as IL6, HA, and sIL2R. An attempt to look at the local glandular inflammatory activity during hydroxychloroquine treatment was make by Kenos et al using Galiun scan. The authors did not observe any decrease in the Galium scanning activity, but it should be noted that only five patients had been investigated by this method. The use of saliva as a diagnostic fluid in SS has been proposed by us and others. ${ }^{5}$ Various markers of inflammation, such as prostaglandin $\mathrm{E}_{2}$, thromboxane $\mathrm{B}_{2}$, IL6, and HA have been reported to be increased in the saliva of SS patients. ${ }^{569}$ The meaning of these markers in the saliva differs and while salivary IL6 concentrations correlated with the histological scoring no such correlation could be detected with salivary HA concentrations. $^{7}$ These probably reflect the activity of the inflammatory process taking place in the exocrine glands of SS patients, which is histologically manifested by CD4 positive lymphocytes infiltrating the salivary glands. ${ }^{1}$ For our study we chose both serum and salivary HA, sIL2R, and IL6 whose serum concentrations have been shown to be a useful measure of disease activity in patients with inflammatory arthritides. ${ }^{11}$ In rheumatoid arthritis patients, measurements of serum HA, sIL2R, and IL6 concentrations were found to be a useful measure of disease activity. ${ }^{12}$ Furthermore, several studies have demonstrated that serum IL6 concentrations are reduced in patients with active rheumatoid arthritis after treatment with intramuscular gold, methotrexate, azathioprine or sulphasalazine. ${ }^{13}$ Similar responses have also been detected in serum sIL2R concentrations of rheumatoid arthritis patients after gold and methotrexate treatment. ${ }^{13}$ Moreover, sIL2R concentrations decreased in patients with erosive osteoarthritis after 12 months of treatment with hydroxychloroquine 200 $\mathrm{mg} /$ day. ${ }^{14}$ The mechanism of action by which hydroxychloroquine affects inflammation in the various rheumatic diseases is not fully understood. It has been suggested that the drug causes interference with macrophage processing of antigens, thus resulting in an interaction with $\mathrm{T}$ cell activation. Recent studies have shown that hydroxychloroquine also affects some in vitro production of some cytokines that play a part in rheumatoid arthritis. These reports have shown that hydroxychloroquine inhibits production of proinflammatory cytokines such as tumour necrosis factor $\alpha$, IL6, and interferon $\gamma$ by peripheral mononuclear cells of RA patients. ${ }^{15}$

This study shows the beneficial in vivo effect of the drug on inflammatory markers in SS patients. After 12 months of hydroxychloroquine treatment, serum IL6 concentrations decreased significantly while no change was noted in serum HA and sIL2R concentrations. Furthermore, the net effect on the salivary inflammatory component was even more impressive, with a significant reduction of both salivary IL6 and HA concentrations. We therefore speculate that the length of time used for hydroxychloroquine treatment in all the earlier studies (up to 24 months) was not sufficient for evaluation of xerostomia in SS. It might be that the local salivary anti-inflammatory effect is just a first step of its activity and the clinical improvement will be evident only later. A hint for this assumption might be the fact that the 
reduction of salivary IL6 and HA concentrations was more pronounced in those patients whose parotid gland was reduced during the following period. On the other hand, even through each patient serves as his own control such a study design cannot rule out the effects of natural remission in the disease process. Therefore, a double blind controlled study of hydroxychloroquine treatment in SS for much longer periods is indicated to evaluate our hypothesis.

1 Skopouli FN, Fox PC, Galanopoulou V, Atkinson JC, Jaffa ES, Moutsopoulos HM. T-cell subpopulation in labia minor salivary gland histopathologic lesion of Sjögren's syndrome. J Rheumatol 1991;18:210-14

2 Fox RI, Chan E, Benton L, Fong S, Friedlaender M, Howell FV. Treatment of primary Sjögren's syndrome with hydroxychloroquine. Am J Med 1988;85 (suppl 4A):62-7

3 Kruize AA, Hene RJ, Kallenberg CGN, van Bijesterveld OP, van der Heide A, Kater L, et al. Hydroxychloroquine treatment for primary Sjögren's syndrome: a two year double blind crossover trial. Ann Rheum Dis 1993;52:360-4.

4 Fox RI, Dixon R, Guarrasi V, Krubel S. Treatment of primary Sjögren's syndrome with hydroxychloroquine: a retrospective open label study. Lupus 1996;5 (suppl 1):S31-6.

5 Tishler M, Yaron I, Shirazi I, Yaron M. Saliva: an additional diagnostic tool in Sjögren's syndrome. Semin Arthritis diagnostic tool in Sjö

6 Grisius M, Bermudez D, Fox PC. Salivary and serum interleukin-6 in Sjögren's syndrome. J Rheumatol 1997;24: 1089-91.
7 Tishler M, Yaron I, Shirazi I, Yaron M. Salivary and serum hyaluronic acid levels in Sjögren's syndrome. Ann Rheum Dis $1998 ; 57: 506-8$

8 Vitali C, Bombardieri S, Moutsopoulos HM, Balestrieri G, Bencivelli W, Bernstein RM, et al. Preliminary criteria for the classification of Sjögren's syndrome. Results of a prospective concerted action supported by the European Community. Arthritis Rheum 1993;36:340-7.

9 Tishler M, Yaron I, Raz A, Meyer FA, Yaron M. Salivary eicosanoid concentration in patients with Sjögren's syndrome. Ann Rheum Dis 1996;55:202-4.

10 Emlen W, Niebur J, Flanders G, Rutledge J. Measurement of serum hyaluronic acid in patients with rheumatoid arthritis: Correlation with disease. J Rheumatol 1996;23: 974-8.

11 Barrera P, Boerbooms A, Janssen E, Sauerwein RW, Gallati $\mathrm{H}$, Mulder J, et al. Circulatory soluble tumor necrosis factor receptors, interleukin-2 receptors, tumor necrosis factor $\alpha$, and interleukin- 6 levels in rheumatoid arthritis. Arthritis $\alpha$, and interleukin-6 levels

12 Madhok R, Crilly A, Wortson J, Capell H. Serum interleukin-6 levels in rheumatoid arthritis: correlation with clinical and laboratory indices of disease activity. Ann Rheum Dis 1993;52:232-4.

13 Crilly A, McInness IB, McDonald AG, Watson J, Capell HA, Madhok R. Interleukin-6 and soluble IL-2 receptor levels in patients with rheumatoid arthritis treated with low dose oral methotrexate. J Rheumatol 1995;22:224-6.

14 Punzi L, Bertazzolo N, Pianon M, Michelotto M, Todesco S. Soluble interleukin-2 receptors and treatment with hydroxychloroquine in erosive osteoarthritis. J Rheumatol 1996;23:1477.

15 Sperber K, Ouraiski H, Kalb TH, Panja A, Stecher V, Mayer L. Selective regulation of cytokine secretion by hydroxychloroquine: Inhibition of interleukin-1 alpha and IL-6 in human monocytes and T cells. J Rheumatol 1993; 20:803. 